Revista de Matemática: Teoría y Aplicaciones 1998 5(1) : 25-37

CIMPA - UCR - CCSS ISSN: 1409-2433

\title{
EL 'UNRAVELLING' PARA BIGRÁFICAS DIFERENCIALES
}

\author{
JuAN BOZA CORDERO*
}

Recibido: 7 Noviembre 1997

\begin{abstract}
Resumen
Se examinan las principales propiedades del algoritmo llamado 'unravelling' para bigráficas diferenciales. Este algoritmo fue originalmente desarrollado para categoras graduadas diferenciales y fue util in la prueba de la celebrada prueba 'tame-wild' del teorema de Drozd. En primer lugar describimos el algoritmo, y luego establecemos en detalle la existencia de una equivalencia entre ciertas subcategoras de representaciones de los bigrafos originales y derivados. También exhibimos el comportamiento preciso de la norma y de la forma cuadrática bajo el algoritmo.
\end{abstract}

Palabras-clave: representación, bigráfica diferencial, 'unravelling', forma cuadrática, equivalencia.

\begin{abstract}
Here are examined the main properties of the algorithm called 'unravelling' for differential bigraphs. This algorithm was originally developed for differential graded categories and was useful in the proof of the celebrated 'tame-wild' theorem of Drozd's. First we describe the algorithm, and then we establish in detail the existence of an equivalence between certain subcategories of representations of the original and the derived bigraphs. We also exhibit the precise behaviour of the norm and the quadratic form under the algorithm.
\end{abstract}

Keywords: representation, differential bigraphic, unravelling, quadratic form, equivalence.

AMS Subject Classification: 16G99

\footnotetext{
*Escuela de Matemática, Universidad de Costa Rica, 2060 San José, Costa Rica
} 


\section{Introducción}

En $\left[\mathrm{Bo}_{1}, \mathrm{Bo}_{2}\right]$ se realizó un estudio de tres algoritmos de reducción para bigráficas diferenciales (regularización, eliminación de objetos, reducción de una flecha), estudio que será completado ahora con la exposición del algoritmo conocido por su nombre inglés como 'unravelling' $\left[\mathrm{CB}_{1}, \mathrm{D}_{1}, \mathrm{D}_{2}\right]$. Se tendrán así a disposición los elementos mínimos necesarios para entender, por ejemplo, la demostración del 'teorema manso-salvaje' de Drozd. Asimismo, el lector tendrá acceso a las aplicaciones novedosas de esta técnica algorítmica al estudio de familias de representaciones de bocses, que se desarrollan en [BB].

El trabajo empieza con una descripción exhaustiva del 'unravelling'. Se construye en detalle la bigráfica diferencial obtenida al aplicar el algoritmo, prestando la atención debida a la definición de la diferencial de las flechas. Se demuestra luego que efectivamente existe una equivalencia entre ciertas subcategorías de las categorías de representaciones de la bigráfica inicial y la derivada, en correspondencia con los resultados clásicos para bocses. Para terminar se expone un ejemplo sencillo, con la idea de que el lector pueda intuir el por qué del 'unravelling'.

Se utilizan libremente la notación y resultados básicos acerca de bigráficas diferenciales de $\left[\mathrm{Bo}_{1}, \mathrm{Bo}_{2}\right] . k$ denota un campo algebraicamente cerrado.

\section{El algoritmo}

Sea $(\mathcal{B}, \delta, k)$ una bigráfica diferencial triangular con vértices $X_{1}, \ldots, X_{t}$ y estratificación $\left(a_{1}, \ldots, a_{n} ; v_{1}, \ldots, v_{m}\right)$. Supóngase que existe un lazo $\alpha$ de grado 0 en $X$, con diferencial nulo : $\delta(\alpha)=0$. Dados $\lambda \in k, r \geq 0$ entero, se define una bigráfica diferencial $\left(\mathcal{B}^{\prime}, \delta^{\prime}, k\right)$ con vértices $Z_{0}, Z_{1}, \ldots, Z_{r}, X_{2}, \ldots, X_{t}$.

Las flechas de grado 0 en $\mathcal{B}^{\prime}$ incluyen un lazo $\alpha^{\prime}$ en $Z_{0}$, y las demás son determinadas por las flechas $a \neq \alpha$ de grado 0 en $\mathcal{B}$, del modo siguiente: el conjunto $\Lambda=$ $\{(i, s): 1 \leq i \leq r, 1 \leq s \leq i\} \cup\{(0,0)\}$ se ordena de la siguiente manera : $(0,0)<(1,1)<$ $(2,1)<(2,2)<(3,1)<(3,2)<(3,3)<\ldots<(r, 1)<(r, 2)<\ldots<(r, r)$. Se escribe : $\beta=(i, s) \in \Lambda, d=1+1+2+3+\ldots+r$. (i) Para toda $a: X_{p} \longrightarrow X_{q}$, con $p, q \neq 1$ , se define una flecha $a: X_{p} \longrightarrow X_{q}$ en $\mathcal{B}^{\prime}$. (ii) Para $a: X_{p} \longrightarrow X_{1}, p \neq 1$, se toman $d$ flechas $a_{\beta 1}=a_{(i, s) 1}: X_{p} \longrightarrow Z_{i}$, en donde $\beta=(i, s) \in \Lambda$. Así, se tienen una flecha $X_{p} \longrightarrow Z_{0}$, e $i$ flechas $X_{p} \longrightarrow Z_{i}(1 \leq i \leq r)$. (iii) Para $a: X_{1} \longrightarrow X_{p}, p \neq 1$, se tienen $d$ flechas $a_{1 \beta}=a_{1(i, s)}: Z_{i} \longrightarrow X_{p},(1 \leq i \leq r)$. (iv) Todo lazo $a \neq \alpha$ en $X_{1}$, determina $d^{2}$ flechas, denotadas por $a_{\beta \gamma}, \beta, \gamma \in \Lambda$, tales que si $\beta=(i, s), \gamma=(j, l)$, entonces $a_{\beta \gamma}=a_{(i, s)(j, l)}: Z_{j} \longrightarrow Z_{i}$.

Las flechas de grado 0 en $\mathcal{B}^{\prime}$ se utilizan para definir matrices $A_{a}$, para $a$ en $\mathcal{B}$, según los anteriores casos, así: (i) $A_{a}=(a)$. (ii) $A_{a}=\left(a_{\beta 1}\right)_{\beta \in \Lambda}$, matriz $d \times 1$ si $a: X_{p} \longrightarrow X_{1}, p \neq 1$; (iii) $A_{a}=\left(a_{1 \beta}\right)_{\beta \in \Lambda}$, matriz $1 \times d$, si $a: X_{1} \longrightarrow X_{p}, p \neq 1$. (iv) $A_{a}=\left(a_{\beta \gamma}\right)_{\beta, \gamma \in \Lambda}$, matriz $d \times d$. (v) $A_{\alpha}$ es la matriz $d \times d$, diagonal por bloques, denotados $B_{00}=\left(\alpha^{\prime}\right), B_{i i}=\left(J_{\lambda}^{i}\right) e_{i}$, para $i \geq 1$, en donde $e_{i}$ denota el idempotente en $Z_{i}, 1 \leq i \leq r$. Esta notación matricial será útil para definir más adelante la diferencial en $\mathcal{B}^{\prime}$.

Las flechas de grado 1 en $\mathcal{B}^{\prime}$ son de dos clases : (i) Para $v: X_{p}--\rightarrow X_{q}$ en $\mathcal{B}, g r(v)=1$, se define una matriz $A_{v}$, por un procedimiento similar al aplicado para construir la matriz 
$A_{a}, g r(a)=0, a \neq \alpha$. Las entradas de $A_{v}=\left(v_{\beta \gamma}\right)$ son las flechas de grado 1 del primer tipo. (ii) Flechas definidas por $\alpha$ : se define una matriz $X^{\prime}=\left(X_{i j}\right)_{1 \leq i, j \leq r}$, de tamaño $d^{\prime} \times d^{\prime},\left(d^{\prime}=1+2+\cdots+r\right)$, en donde $X_{i j}$ es un bloque con $i$ filas y $j$ columnas, del siguiente estilo:

○ si $i=j$,

$$
X_{i i}=\left(\begin{array}{ccccc}
x_{i i}^{1} & x_{i i}^{2} & x_{i i}^{3} & \cdots & x_{i i}^{i} \\
0 & x_{i i}^{1} & x_{i i}^{2} & \ddots & \\
\vdots & 0 & \ddots & \ddots & \\
& \vdots & \ddots & \ddots & \\
0 & 0 & 0 & \cdots & x_{i i}^{1}
\end{array}\right) \text {, }
$$

para ciertos $x_{i j}^{l}, 1 \leq l \leq i$ (así, $X_{i i}$ posee $i$ distintos elementos en sus entradas);

○ si $i<j, j=i+t, X_{i j}=X_{i, i+t}$ tiene nulas sus primeras $t$ columnas, y es de la forma $: X_{i j}=\left((0, \ldots, 0), X_{i i}^{(i j)}\right)$, con $X_{i i}^{(i j)}$ bloque $i \times i$ del tipo $X_{i i}$ anteriormente introducido;

○ si $i>j, i=j+t, X_{i j}=X_{j+t, j}$ tiene nulas sus últimas $t$ filas, y es de la forma $\left(X_{j j}^{(i j)},(0, \cdots, 0)\right)^{t r}$, con $X_{j j}^{(i j)}$ bloque $j \times j$ del tipo $X_{j j}$ anterior.

Por definición, las flechas de grado 1 en $\mathcal{B}^{\prime}$ determinadas por $\alpha$, se encuentran en correspondencia biyectiva con las entradas no nulas, distintas entre sí, y fuera de la diagonal principal de la matriz $X^{\prime}$ (o sea, no se toman en cuenta $x_{(11)(11)}, x_{(21)(21)}, x_{(22)(22)}, \ldots$ ). Estas flechas se denotan por las mismas letras de las entradas de $X^{\prime}$, y su orientación es como sigue :

○ el bloque $X_{i i}$ determina $i-1$ lazos $: x_{(i, 1)(i, 2)}, \ldots x_{(i, 1)(i, i)}: Z_{i}--\rightarrow Z_{i}$;

○ para $i<j=i+t$, se tienen $i$ flechas : $x_{(i, 1)(j, t+1)}, \ldots, x_{(i, 1)(j, j)}: Z_{j}--\rightarrow Z_{i}$;

$\circ$ para $j<i=j+t$, se tienen $j$ flechas : $x_{(i, 1)(j, 1)}, \ldots, x_{(i, 1)(j, j)}: Z_{i}--\rightarrow Z_{j}$.

Con esto termina la descripción de las flechas de $\mathcal{B}^{\prime}$.

Con la matriz $X^{\prime}$ se define una nueva matriz $X$, diagonal por bloques, de tamaño $d \times d, d=1+d^{\prime}$, con su primer bloque nulo y el segundo igual a $X^{\prime}$. Tomando $J=$ $J_{\lambda}^{1} \oplus J_{\lambda}^{2} \oplus \cdots \oplus J_{\lambda}^{r}$, la matriz $X^{\prime}$ verifica la importante relación : $X^{\prime} J=J X^{\prime}$. De hecho, la última igualdad caracteriza a $X^{\prime}[\mathrm{Ma}, \S 16.6]$.

Ahora se procede a definir la diferencial $\delta^{\prime}$ en $\mathcal{B}^{\prime}$, de manera semejante a como se hizo para el caso de la reducción de una flecha $\left[\mathrm{Bo}_{1}\right.$, Cap. $\left.2, \S 4 ; \mathrm{Bo}_{2}, \S 4,3\right]$. Para todo vértice $X_{p}$ de $\mathcal{B}$, se define una matriz $Y_{p}$ así: $Y_{p}=0$, si $p \neq 1, Y_{1}$ es la matriz obtenida de la matriz $X$ al tomar nulas en ésta todas las entradas de la diagonal principal. Para toda flecha $h$ de $X_{p}$ en $X_{q}$ en $\mathcal{B}$, las diferenciales de las flechas que ella determina en $\mathcal{B}^{\prime}$, mismas que aparecen como las entradas de la matriz $A_{h}$, se definen como las entradas de la matriz $\delta^{\prime}\left(A_{h}\right)$, en donde

$$
\delta^{\prime}\left(A_{h}\right)=Y_{q} A_{h}-(-1)^{\mathrm{gr} h} A_{h} Y_{p}+A_{\delta(h)} ; \delta^{\prime}\left(A_{\alpha}\right)=0 ; \delta^{\prime}\left(Y_{l}\right)=Y_{l}^{2} .
$$

La comprobación de que $\delta^{\prime}$ es efectivamente una diferencial es semejante al caso de la reducción de la flecha, y no se hace aquí. Consecuencias inmediatas de la definición : $\delta^{\prime}\left(\alpha^{\prime}\right)=0, \delta^{\prime}\left(e_{i}\right)=0$. 


\section{La equivalencia}

Es fundamental la existencia de un funtor $F: \operatorname{rep\mathcal {B}^{\prime }} \longrightarrow$ rep $\mathcal{B}$, que se construye a continuación.

Si se tiene un objeto $N \in \operatorname{rep\mathcal {B}^{\prime }}$, se define $M=F(N) \in \operatorname{rep\mathcal {B}}$, poniendo : $M\left(X_{p}\right)=$ $N\left(X_{p}\right)$, si $p \neq 1 ; M\left(X_{1}\right)=N\left(Z_{0}\right) \oplus \bigoplus_{i=1}^{r} N\left(Z_{i}\right)^{(i)}$, en donde $N\left(Z_{i}\right)^{(i)}=N\left(Z_{i}\right) \oplus \cdots \oplus N\left(Z_{i}\right)$, $i$ veces. Para definir $M$ en las flechas de grado 0 , sea $a: X_{p} \longrightarrow X_{q}$ una flecha en $\mathcal{B}$. Si $p, q \neq 1$, se pone $M(a)=N(a)$. Si $a: X_{p} \longrightarrow X_{1}, p \neq 1, M(a)$ es la matriz columna con $d$ bloques : $M(a)=\left(N\left(a_{\beta 1}\right)\right)_{\beta \in \Lambda}$. Similarmente, para $a: X_{1} \longrightarrow X_{p}, p \neq 1$, se define $M(a)=\left(N\left(a_{1 \beta}\right)\right)_{\beta \in \Lambda}$. Para un lazo $a: X_{1} \longrightarrow X_{1}, a \neq \alpha$, se define $M(a)=$ $\left(N\left(a_{\beta \gamma}\right)\right)_{\beta, \gamma \in \Lambda}$. Finalmente, $M(\alpha): M_{1} \longrightarrow M_{1}$ es la suma directa de matrices : $M(\alpha)=$ $N\left(\alpha^{\prime}\right) \oplus \bar{J}_{\lambda}^{1} \oplus \ldots \oplus \bar{J}_{\lambda}^{r}$, en donde $\bar{J}_{\lambda}^{i}$ es la matriz de bloques $\bar{J}_{\lambda}^{i}=J_{\lambda} i d_{N\left(Z_{i}\right)},(1 \leq i \leq r)$.

Para definir $F$ en morfismos, sea $f: N \longrightarrow N^{\prime}$ un morfismo en $\mathcal{B}^{\prime}$, dado por $f=$ $\left(f_{0}^{0}, \ldots, f_{r}^{0}, \varphi_{2}^{0}, \ldots, \varphi_{t}^{0} ; f^{1}(v), g r(v)=1\right)$. Se define

$g=\left(g_{i}^{0}, 1 \leq i \leq t ; g^{1}\left(v_{j}\right), 1 \leq j \leq m\right)$, tomando $g_{1}^{0}$ como la matriz $d \times d$ :

$$
g_{1}^{0}=\left(\begin{array}{cc}
f_{0}^{0} & 0 \\
0 & X^{\prime \prime}
\end{array}\right)
$$

en donde la matriz $X^{\prime \prime}:=\left(X_{\beta \gamma}^{\prime \prime}\right)_{\beta, \gamma \in \Lambda-\{(0,0)\}}$ tiene entradas $X_{(i, 1)(i, 1)}^{\prime \prime}=X_{(i, 2)(i, 2)}^{\prime \prime}=\ldots=$ $X_{(i, i)(i, i)}^{\prime \prime}=f_{i}^{0}, i=1, \ldots, r ; X_{\beta \gamma}^{\prime \prime}=f^{1}\left(x_{\beta \gamma}\right)$, para $(\beta, \gamma)$ fuera de la diagonal principal ; $X_{\beta \gamma}^{\prime \prime}=0$, si no está definida la flecha $x_{\beta \gamma}$ en $\mathcal{B}^{\prime}$. Se adoptará una notación especial para los bloques $X_{i i}^{\prime \prime}$ de la matriz $X^{\prime \prime}$, escribiendo:

$$
X_{i i}^{\prime \prime}=\left(\begin{array}{llllll}
f_{i}^{0} & f^{1}\left(x_{i i}^{2}\right) & . & . & . & f^{1}\left(x_{i i}^{i}\right) \\
0 & f_{i}^{0} & f^{1}\left(x_{i i}^{2}\right) & \cdot & . & \cdot \\
\cdot & 0 & \cdot & . & . & \cdot \\
\cdot & \cdot & . & . & \cdot & \cdot \\
\cdot & \cdot & . & 0 & f_{i}^{0} & f^{1}\left(x_{i i}^{2}\right) \\
0 & \cdot & . & . & 0 & f_{i}^{0}
\end{array}\right) .
$$

Lema $g: M=F(N) \longrightarrow F\left(N^{\prime}\right)=M^{\prime}$ es morfismo.

Demostración Sea $a: X_{p} \longrightarrow X_{q}$ en $\mathcal{B}$, se consideran dos casos: (i) $a=\alpha, \delta(a)=$ $\delta(\alpha)=0$; sea $\bar{J}=\bar{J}_{\lambda}^{1} \oplus \ldots \oplus \bar{J}_{\lambda}^{r}$. Por la escogencia de $X^{\prime \prime}, X^{\prime \prime} \bar{J}=\bar{J} X^{\prime \prime}$, y de $\delta^{\prime}\left(\alpha^{\prime}\right)=0$ se sigue : $f_{0}^{0} N\left(\alpha^{\prime}\right)=N^{\prime}\left(\alpha^{\prime}\right) f_{0}^{0}$. Luego $g_{1}^{0} M(\alpha)=M^{\prime}(\alpha) g_{1}^{0}$. (ii) Si $a \neq \alpha$, se procede como en $\left[\mathrm{Bo}_{1}\right.$, Cap. 2, 4.6]. Se ha demostrado entonces que $g$ es morfismo.

Fácilmente se demuestra también que $F$ es un funtor.

Se denota ahora por $\operatorname{rep}_{(\lambda, r)}$ la subcategoría plena de $\operatorname{rep} \mathcal{B}$, formada por las representaciones $M$ de $\mathcal{B}$, tales que en la forma canónica de Jordan de $M(\alpha)$, el mayor $\lambda$-bloque tiene dimensión a lo sumo $r$. Asimismo, $\operatorname{rep\mathcal {B}^{\prime }}{ }_{\left(Z_{0}, x-\lambda\right)}$ es la subcategoría plena de $r e p \mathcal{B}^{\prime}$ constituida por las representaciones $N$, tales que $N\left(\alpha^{\prime}\right)-\lambda I$ es invertible, i.e. $\lambda$ no es valor propio de $N\left(\alpha^{\prime}\right)$.

Teorema 1 El funtor $F:$ rep $\mathcal{B}^{\prime} \longrightarrow$ rep $\mathcal{B}$ induce una equivalencia 


$$
F: \operatorname{rep\mathcal {B}_{(Z,x-\lambda )}^{\prime }} \stackrel{\sim}{\longrightarrow} \operatorname{ep} \mathcal{B}_{(\lambda, r)}
$$

Demostración (i) $F$ es denso. En efecto, sea $M \in \operatorname{rep} \mathcal{B}_{(\lambda, r)}$, entonces la forma canónica de $M(\alpha)$ puede escribirse como $M(\alpha)_{\text {can }}=L \oplus n_{1} J_{\lambda}^{1} \oplus \ldots \oplus n_{r} J_{\lambda}^{r}$, con $L_{l \times l}$, tal que $\lambda$ no es valor propio de $L$, y $n_{1}, \ldots, n_{r}$ enteros $\geq 0$. Se tiene $: M_{1}=k^{l} \oplus k^{n_{1}} \oplus k^{2 n_{2}} \oplus \ldots \oplus k^{r n_{r}}$. Se define una representación $N$ de $\mathcal{B}^{\prime}$, tal que $N\left(Z_{0}\right)=k^{l}, N\left(Z_{i}\right)=k^{n_{i}}, i=1,2, \ldots r ; N\left(X_{i}\right)=$ $M\left(X_{i}\right), i=2, \ldots, t$.

La matriz $N(b)$, con $b$ flecha de grado 0 en $\mathcal{B}^{\prime}$, se define por casos : $N\left(\alpha^{\prime}\right)=L$; si $a: X_{p} \longrightarrow X_{q}$ en $\mathcal{B},(p, q \neq 1), N(a)=M(a)$; si $a: X_{1} \longrightarrow X_{1}, a \neq \alpha$, se escribe $M_{1}=\bigoplus_{\beta \in \Lambda} V_{\beta}$, con $V_{(0,0)}=k^{l}, V_{(i, s)}=k^{n_{i}}$, y entonces con respecto a esta descomposición de $M_{1}, M(a)$ es una matriz de bloques : $M(a)=\left(M(a)_{\beta \gamma}\right)$, con $M_{\beta \gamma}=M_{(i, s)(j, l)}: V_{\gamma}=$ $V_{(j, l)} \longrightarrow V_{\beta}=V_{(i, s)}$. Para la flecha $a_{\beta \gamma}=a_{(i, s)(j, l)}: Z_{j} \longrightarrow Z_{i}$, se toma $: N\left(a_{\beta \gamma}\right)=T_{\beta \gamma}$. Se procede de manera semejante para definir $N$ en las flechas que provienen de flechas $X_{1} \longrightarrow X_{q}, X_{q} \longrightarrow X_{1}, q \neq 1$.

La misma escogencia de $N\left(\alpha^{\prime}\right)=L$ garantiza que $N \in \operatorname{rep} \mathcal{B}_{\left(Z_{0}, x-\lambda\right)}^{\prime}$. Además, para el funtor $F$ antes definido, la representación $F(N)$ verifica $F(N)\left(X_{1}\right)=M\left(X_{1}\right), F(N)\left(X_{i}\right)=$ $N\left(X_{i}\right)=M\left(X_{i}\right),(i=2, \ldots, t), F(N)(\alpha)=N\left(\alpha^{\prime}\right) \oplus \bar{J}=L \oplus \bar{J}=M(\alpha)_{\text {can }}$, (aquí $\bar{J}=$ $\left.n_{1} J_{\lambda}^{1} \oplus \ldots \oplus n_{r} J_{\lambda}^{r}\right)$, de donde $F(N) \cong M$.

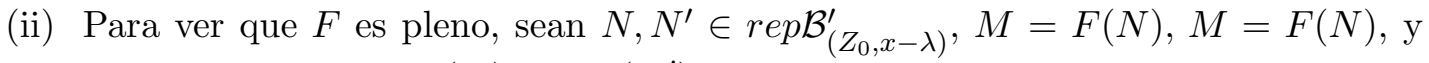
considérese un morfismo $g: F(N) \longrightarrow F\left(N^{\prime}\right)$, dado por

$\left(g_{i}^{0}, i=1, \ldots, t ; g^{1}\left(v_{s}\right), s=1, \ldots, m\right)$. Se define $f: N \longrightarrow N^{\prime}$, tal que $g=F(f)$, de la siguiente manera : como $\delta(\alpha)=0$, se tiene $g_{1}^{0} M(\alpha)=M^{\prime}(\alpha) g_{1}^{0}$. Además, $M(\alpha)=$ $N\left(\alpha^{\prime}\right)_{l \times l} \oplus n_{1} J_{\lambda}^{1} \oplus n_{2} J_{\lambda}^{2} \oplus \ldots \oplus n_{r} J_{\lambda}, M^{\prime}(\alpha)=N^{\prime}\left(\alpha^{\prime}\right)_{t \times t} \oplus m_{1} J_{\lambda}^{1} \oplus m_{2} J_{\lambda}^{2} \oplus \ldots \oplus m_{r} J_{\lambda}^{r}$.

Sean $\bar{J}=n_{1} J_{\lambda}^{1} \oplus n_{2} J_{\lambda}^{2} \oplus \ldots \oplus n_{r} J_{\lambda}^{r}, \bar{J}^{\prime}=m_{1} J_{\lambda}^{1} \oplus m_{2} J_{\lambda}^{2} \oplus \ldots \oplus m_{r} J_{\lambda}^{r}$. De la igualdad de matrices : $g_{1}^{0}\left(\begin{array}{cc}L & 0 \\ 0 & J\end{array}\right)=\left(\begin{array}{cc}L^{\prime} & 0 \\ 0 & J^{\prime}\end{array}\right) g_{1}^{0}$, y del hecho de que $\lambda$ no es valor propio de $N\left(\alpha^{\prime}\right)$ ni de $N^{\prime}\left(\alpha^{\prime}\right)$, se deduce que $g_{1}^{0}$ tiene una expresión matricial $g_{1}^{0}=\left(\begin{array}{cc}f_{0}^{0} & 0 \\ 0 & Q\end{array}\right)$, con $f_{0}^{0}$ de tamaño $t \times l$. De la igualdad : $Q \bar{J}=\bar{J}^{\prime} Q$ se colige que la matriz $Q$ posee una estructura igual a la de la matriz anteriormente denotada por $X^{\prime \prime}$.

Para $i=1, \ldots, r$, se toma $f_{i}^{0}$ como el valor común de la diagonal del bloque $X_{i i}$ de la matriz $g_{1}^{0}$. Para $i=2, \ldots, t, f_{i}^{0}=g_{i}^{0}$.

Para las flechas de grado 1 en $\mathcal{B}^{\prime}$ que provienen de $\alpha$, y que han sido denotadas por $x_{\beta \gamma}$ (para ciertos $\beta, \gamma \in \Lambda$ ), se toma $f^{1}\left(x_{\beta \gamma}\right)=\left(g_{0}^{1}\right)_{\beta \gamma}=Q_{\beta \gamma}$. Para $v_{s}: X_{p}-\rightarrow X_{q}$ en $\mathcal{B}$, la matriz $g^{1}\left(v_{s}\right)$ se define en componentes como $g^{1}\left(v_{s}\right)=\left(g^{1}\left(v_{i j}^{(s)}\right)\right)$, lo cual permite definir : $f^{1}\left(v_{i j}^{(s)}\right)=g^{1}\left(v_{i j}^{(s)}\right)$.

Se ha construido así $f=\left(f_{0}^{0}, \ldots, f_{0}^{0}, f_{2}^{0}, \ldots, f_{t}^{0} ; f^{1}\left(x_{\beta \gamma}\right), f^{1}\left(v_{i j}^{(s)}\right)\right)$, que determina un morfismo $f: N \longrightarrow N^{\prime}$ en $\mathcal{B}^{\prime}$. Es claro que $g=F(f)$.

Finalmente, es fácil ver que el funtor $F$ es fiel. 


\section{La norma y la forma cuadrática}

Es importante tener control sobre el comportamiento de la norma y la forma cuadrática frente a los algoritmos, como se vio ya en $\left[\mathrm{Bo}_{1}, \mathrm{Bo}_{2}\right]$ para los otros algoritmos. A continuación se detalla lo que sucede con el 'unravelling'.

Teorema 2 En las condiciones del teorema anterior, sea $M \cong F(N)$, en donde $\underline{\operatorname{dim}} N=$ $\left(z_{0}, z_{1}, \ldots, z_{r} ; x_{2}, \ldots, x_{t}\right)$. Entonces $\underline{\operatorname{dim} M}=\left(z_{0}+\sum_{i=1}^{r} i z_{i}, x_{2}, \ldots, x_{t}\right)$, y para las normas se verifica : $\|N\|=\|M\|-\left(\left(\operatorname{dim} M_{1}\right)^{2}-z_{0}^{2}\right) \leq\|M\|$. En particular, si $\lambda$ es valor propio de $M(\alpha)$, la desigualdad es estricta.

Demostración Sólo resta demostrar la segunda afirmación. Si $m_{i j}$ es el número de flechas de grado 0 entre $i$ y $j$ en $\mathcal{B}$, entonces :

$$
\|M\|=m_{11}\left(z_{0+} \sum_{i=1}^{r} i z_{i}\right)^{2}+\sum_{p=2}^{t} m_{1 p}\left(z_{0}+\sum_{i=1}^{r} i z_{i}\right) x_{p}+\sum_{p, q=2}^{t} m_{p q} x_{p} x_{q} .
$$

Según la definición de $\mathcal{B}^{\prime}$ se tiene :

$$
\begin{aligned}
\|N\|= & z_{0}^{2}+\left(m_{11}-1\right)\left[z_{0}^{2}+\sum_{i=1}^{r} i^{2} z_{i}^{2}+\sum_{i=1}^{r} 2 i z_{0} z_{i}+\sum_{i, j=1, i<j}^{r} i j z_{i} z_{j}\right] \\
& +\sum_{p=2}^{t} m_{1 p}\left(z_{0} x_{p}+\sum_{i=1}^{r} i z_{i} x_{p}\right)+\sum_{p, q=2}^{t} m_{p q} x_{p} x_{q} \\
= & z_{0}^{2}+\left(m_{11}-1\right)\left(z_{0+} \sum_{i=1}^{r} i z_{i}\right)^{2}+\sum_{p=2}^{t} m_{1 p}\left(z_{0+} \sum_{i=1}^{r} i z_{i}\right) \\
& +\sum_{p, q=2 t} m_{p q} x_{p} x_{q} .
\end{aligned}
$$

Un cálculo sencillo conduce a :

$$
\|M\|-\|N\|=\left(z_{0}+\sum_{i=1}^{r} i z_{i}\right)^{2}-z_{0}^{2}=\left(\operatorname{dim} M_{1}\right)^{2}-z_{0}^{2},
$$

y de aquí : $\|N\|=\|M\|-\left(\left(\operatorname{dim} M_{1}\right)^{2}-z_{0}^{2}\right)$. La última afirmación del teorema se sigue de :

$$
\left(\operatorname{dim} M_{1}\right)^{2}-z_{0}^{2}=\sum_{i=1}^{r} i^{2} z_{i}^{2}+\sum_{i=1}^{r} 2 i z_{0} z_{i}+\sum_{i, j=1, i<j}^{r} i j z_{i} z_{j} .
$$

Teorema 3 En las condiciones del teorema anterior, para $F(N)=M$ se verifica:

$$
q_{\mathcal{B}^{\prime}}(\underline{\operatorname{dim}} N)-q_{\mathcal{B}}(\underline{\operatorname{dim}} M)=\sum_{i=1}^{r} i z_{i}^{2}+2 \sum_{i, j=1 ; i<j}^{r} i z_{i} z_{j}
$$


Demostración Para $M$ se tiene :

$$
q_{\mathcal{B}}(\underline{\operatorname{dim}} M)=\left(z_{0}+\sum_{i=1}^{r} i z_{i}\right)^{2}+\sum_{p=2}^{t} x_{p}^{2}-\|M\|+q_{\mathcal{B}}^{(1)}(\underline{\operatorname{dim}} M),
$$

y para $N$ vale :

$$
q_{\mathcal{B}^{\prime}}(\underline{\operatorname{dim}} N)=\sum_{i=0}^{r} z_{i}^{2}+\sum_{p=2}^{t} x_{p}^{2}-\|N\|+q_{\mathcal{B}^{\prime}}^{(1)}(\underline{\operatorname{dim}} N),
$$

en donde :

$$
\begin{aligned}
q_{\mathcal{B}}^{(1)}(\underline{\operatorname{dim}} M)= & n_{11}\left(x_{1}+x_{3}\right)^{2}+n_{22}\left(x_{3}+x_{2}\right)^{2} \\
& +n_{12}\left(x_{1}+x_{3}\right)\left(x_{3}+x_{2}\right) \\
& +\sum_{p=4}^{n} n_{1 p}\left(x_{1}++x_{3}\right) x_{p}+\sum_{p=4}^{n} n_{2 p}\left(x_{3}+x_{2}\right) x_{p} \\
& +\sum_{p, q=4}^{n} n_{p q} x_{p} x_{q} ; \\
q_{\mathcal{B}^{\prime}}^{(1)}(\underline{\operatorname{dim}} N)= & x_{1} x_{3}+x_{3} x_{2}+n_{12}\left(x_{1} x_{2}+x_{1} x_{3}+x_{3} x_{2}+x_{3}^{2}\right) \\
& +n_{11}\left(x_{1}^{2}+2 x_{1} x_{3}+x_{3}^{2}\right)+n_{22}\left(x_{3}^{2}+2 x_{3} x_{2}+x_{2}^{2}\right) \\
& +\sum_{p=4}^{n} n_{1 p}\left(x_{1} x_{p}+x_{3} x_{p}\right)+\sum_{2 p}^{n} n_{2 p}\left(x_{3} x_{p}+x_{2} x_{p}\right) \\
& +\sum_{p, q=4}^{n} n_{p q} x_{p} x_{q} .
\end{aligned}
$$

Se tiene entonces :

$$
\begin{aligned}
q_{\mathcal{B}^{\prime}}(\underline{\operatorname{dim}} N)-q_{B}(\underline{\operatorname{dim}} M)= & {\left[\sum_{i=0}^{r} z_{i}^{2}-\left(z_{0}+\sum_{i=1}^{r} i z_{i}\right)^{2}\right]+\left[\left(z_{0}+\sum_{i=1}^{r} i z_{i}\right)^{2}-z_{0}^{2}\right] } \\
& +\left[\sum_{i=2}^{r}(i-1) z_{i}^{2}+\sum_{i, j=1 ; i<j}^{r} 2 i z_{i} z_{j}\right] \\
= & \sum_{i=1}^{r} z_{i}^{2}+\sum_{i=2}^{r}(i-1) z_{i}^{2}+\sum_{i, j=1 ; i<j}^{r} 2 i z_{i} z_{j} \\
= & z_{1}^{2}+\sum_{i=2}^{r} i z_{i}^{2}+\sum_{i, j=1 ; i<j}^{r} 2 i z_{i} z_{j} \\
= & \sum_{i=1}^{r} i z_{i}^{2}+\sum_{i, j=1 ; i<j}^{r} 2 i z_{i} z_{j} . \text {. }
\end{aligned}
$$




\section{Un ejemplo}

Sea $(\mathcal{B}, \delta, k)$ la bigráfica con un solo vértice $X_{1}$, dos lazos $\alpha, a$ de grado 0 , y un lazo $z$ de grado 1. Supóngase además la diferencial dada por : $\delta(\alpha)=\delta(z)=0, \delta(a)=z \alpha$.

Se realizará el "unravelling" de $\alpha$, tomando $r=2, \lambda \in k$. Como la idea es penetrar un tanto en el por qué del 'unravelling', no se adopta desde el principio el enfoque abstracto desarrollado en páginas anteriores. Más bien se intenta avanzar tanto como sea posible por un camino intuitivo.

El proceso de construir la nueva bigráfica incluye, como se verá, la definición simultánea del funtor $F$.

Sea $M \in r e p \mathcal{B}$, supóngase que $\lambda$ es valor propio de $M(\alpha)$ y para fijar ideas, asúmase que el mayor bloque de Jordan de la forma canónica de $M(\alpha)$ es de orden 2, y que $M(\alpha)_{\text {can }}=L \oplus J_{\lambda}^{1} \oplus J_{\lambda}^{2}$, en donde $L$ es una matriz $l \times l$, tal que $\lambda$ no es valor propio de $L$. Sea $M^{\prime}$ otra representación, tal que $M^{\prime}(\alpha)_{\text {can }}=L^{\prime} \oplus J_{\lambda}^{1} \oplus J_{\lambda}^{2}$, con $L^{\prime}$ una matriz $d \times d$, tal que $\lambda$ no es valor propio de $L^{\prime}$. Sea $g: M \longrightarrow M^{\prime}, g=\left(g_{1}^{0} ; g^{1}(z)\right)$. Se tienen descomposiciones : $M_{1}=k^{l} \oplus k \oplus k^{2}, M_{1}^{\prime}=k^{d} \oplus k \oplus k^{2}$, con respecto a las cuales : $g_{1}^{0}=\left(\begin{array}{cc}P & B \\ C & D\end{array}\right)$, con bloques de dimensiones $P_{d \times l}, B_{d \times 3}, C_{3 \times l}, D_{3 \times 3}$. Obsérvese que $B=0, C=0$. (Se mostrará que $B=0$, la otra afirmación es semejante : $B$ es $2 \times 3$; los vectores propios de $J=J_{\lambda}^{1} \oplus J_{\lambda}^{2}$ son los $v=\left(x_{1}, x_{2}, 0\right)^{t r}$, con $x_{1}, x_{2} \in k$. De $B J=L^{\prime} B$, se sigue que $\lambda(B v)=L^{\prime}(B v)$, y de aquí, $\lambda=0$ ó $B v=0$. De $B v=0, B=\left(\begin{array}{ccc}0 & 0 & b_{13} \\ 0 & 0 & b_{23}\end{array}\right)$, luego $B J=\left(\begin{array}{ccc}0 & 0 & \lambda b_{13} \\ 0 & 0 & \lambda b_{23}\end{array}\right)$. Pero $B J=L^{\prime} B$, así $L^{\prime} B=\lambda B$. Entonces para todo vector $w, L^{\prime}(B w)=\lambda(B w)$. Por hipótesis, $\lambda$ no es valor propio de $L^{\prime}$, de modo que $B w=0$, de donde $B=0)$.

Consecuencia inmediata de $J D=D J$ es que la matriz $D$ tiene la forma especial

$$
D=\left(\begin{array}{lll}
d_{11} & 0 & d_{13} \\
d_{21} & d_{22} & d_{23} \\
0 & 0 & d_{22}
\end{array}\right)
$$

Por razones que se entenderán en un momento, se introduce la notación : $P=f_{0}^{0}, d_{11}=$ $f_{1}^{0}, d_{22}=f_{2}^{0}, d_{13}=x_{13}, d_{21}=x_{21}, d_{23}=x_{23}$. Se tiene entonces

$$
g_{1}^{0}=\left(\begin{array}{llll}
f_{0}^{0} & 0 & 0 & 0 \\
0 & f_{1}^{0} & 0 & x_{13} \\
0 & x_{21} & f_{2}^{0} & x_{23} \\
0 & 0 & & f_{2}^{0}
\end{array}\right)
$$

La transformación lineal $M(a): M_{1} \longrightarrow M_{1}$ se escribe como matriz de bloques (en correspondencia con las descomposiciones de $M_{1}, M_{1}^{\prime}$ ), cada uno de ellos con entradas 
escalares $a_{i j}, 0 \leq i, j \leq 3$, de la siguiente manera :

$$
M(a)=\left(\begin{array}{lll}
a_{00} & a_{01} & \left(a_{02}, a_{03}\right) \\
a_{10} & a_{11} & \left(a_{12}, a_{13}\right) \\
\left(\begin{array}{l}
a_{20} \\
a_{30}
\end{array}\right) & \left(\begin{array}{l}
a_{21} \\
a_{31}
\end{array}\right) & \left(\begin{array}{ll}
a_{22} & a_{23} \\
a_{32} & a_{33}
\end{array}\right)
\end{array}\right)
$$

Se escribirá : $M^{\prime}(a)=\left(a_{i j}^{\prime}\right)$, también en bloques.

Los bloques de $M(a)$ proveen 16 transformaciones lineales, una por cada entrada escalar, tomando: $\left(a_{00}, a_{03}\right)=\left(a_{00}, 0\right)+\left(0, a_{03}\right)$, etc. Se escribirá $: a_{00}:=\left(a_{00}, 0\right), a_{03}:=$ $\left(0, a_{03}\right)$, etc.

Se define ahora una nueva bigráfica $\mathcal{B}^{\prime}$, con 3 vértices : $X_{0}, X_{1}, X_{2}$. En ella se toman 3 flechas de grado $1: x_{13}: X_{2}-\rightarrow X_{1}, x_{21}: X_{1}-\rightarrow X_{2}, x_{23}: X_{2}-\rightarrow X_{2}$; se toman además 16 flechas de grado 1 , denotadas $z_{i j} 0 \leq i, j \leq 3$, y orientadas así : $z_{00}: X_{0}-\rightarrow X_{0}, z_{01}: X_{1}--\rightarrow X_{0}$

$z_{02}, z_{03}: X_{2}--\rightarrow X_{0}, z_{10}: X_{0}-\rightarrow \rightarrow X_{1}, z_{11}: X_{1}--\rightarrow X_{1}, z_{12}, z_{13}: X_{2}--\rightarrow$ $X_{1}, z_{20}, z_{30}: X_{0}--\rightarrow X_{2}, z_{21}, z_{31}: X_{1}--\rightarrow X_{2}, z_{22}, z_{32}, z_{23}, z_{33}: X_{2}-\rightarrow X_{2}$. Las flechas de grado 0 en $\mathcal{B}^{\prime}$ se toman en correspondencia con las 16 entradas de la matriz $M(a)=\left(a_{i j}\right)$, y se denotan también por $a_{i j}$, teniendo $a_{i j}$ la misma orientación que $z_{i j}$. Se añade un lazo $\alpha^{\prime}$ de grado 0 en $X_{0}$.

El siguiente paso es determinar las diferenciales de las flechas en $\mathcal{B}^{\prime}$. Para empezar con las de grado 0 , ya que la diferencial $\delta(a)=z \alpha$, se tiene :

$$
g_{1}^{0} M(a)-M^{\prime}(a) g_{1}^{0}=g^{1}(z) M(\alpha), \operatorname{con} M(\alpha):=\left(\begin{array}{llll}
\alpha^{\prime} & 0 & 0 & 0 \\
0 & \lambda e_{1} & 0 & 0 \\
0 & 0 & \lambda e_{2} & e_{2} \\
0 & 0 & 0 & \lambda e_{2}
\end{array}\right)
$$

Abusando de la notación, se escribe la matriz $g^{1}(z)=\left(z_{i j}\right)$. Al efectuar las operaciones matriciales anteriores y despejar de manera adecuada, es decir, con apego a los fundamentos de la teoría de representaciones de las bigráficas diferenciales [Ro, $\S 4$ : $\left.\mathcal{D}(\alpha)=\alpha u_{a}-u_{b} \alpha\right]$, se llega a la igualdad matricial :

$$
\left(\begin{array}{cccc}
f_{0}^{0} a_{00}-a_{00}^{\prime} f_{0}^{0} & f_{0}^{0} a_{01}-a_{01}^{\prime} f_{1}^{0} & f_{0}^{0} a_{02}-a_{02}^{\prime} f_{2}^{0} & f_{0}^{0} a_{03}-a_{03}^{\prime} f_{2}^{0} \\
f_{1}^{0} a_{10}-a_{10}^{\prime} f_{0}^{0} & f_{1}^{0} a_{11}-a_{11}^{\prime} f_{1}^{0} & f_{1}^{0} a_{12}-a_{12}^{\prime} f_{2}^{0} & f_{1}^{0} a_{13}-a_{13}^{\prime} f_{2}^{0} \\
f_{2}^{0} a_{20}-a_{20}^{\prime} f_{0}^{0} & f_{2}^{0} a_{21}-a_{21}^{\prime} f_{1}^{0} & f_{2}^{0} a_{22}-a_{22}^{\prime} f_{2}^{0} & f_{2}^{0} a_{23}-a_{23}^{\prime} f_{2}^{0} \\
f_{2}^{0} a_{30}-a_{30}^{\prime} f_{0}^{0} & f_{2}^{0} a_{31}-a_{31}^{\prime} f_{1}^{0} & f_{2}^{0} a_{32}-a_{32}^{\prime} f_{2}^{0} & f_{2}^{0} a_{33}-a_{33}^{\prime} f_{2}^{0}
\end{array}\right)=
$$




$$
=\left(\begin{array}{llll}
z_{00} L & \lambda z_{01}+a_{02}^{\prime} x_{21} & \lambda z_{02} & z_{02}+\lambda z_{03} \\
& & & +a_{01}^{\prime} x_{13}+a_{02}^{\prime} x_{23} \\
& & & \\
z_{10} L-x_{13} a_{30} & \lambda z_{11}+a_{12}^{\prime} x_{21} & \lambda z_{12}-x_{13} a_{31} & z_{12}+\lambda z_{13} \\
& -x_{13} a_{31} & & a_{11}^{\prime} x_{13}+a_{12}^{\prime} x_{23} \\
& & & -x_{13} a_{33} \\
z_{20} L-x_{21} a_{10} & \lambda z_{21}-x_{21} a_{11} & \lambda z_{22}-x_{21} a_{12} & z_{22}+\lambda z_{23} \\
-x_{23} a_{30} & x_{23} a_{31} & -x_{23} a_{32} & a_{21}^{\prime} x_{13}+a_{22}^{\prime} x_{23} \\
& & & -x_{21} a_{13}-x_{23} a_{33} \\
& & & \\
z_{30} L & \lambda z_{31}+a_{32}^{\prime} x_{21} & \lambda z_{32} & z_{32}+\lambda z_{33} \\
& & & a_{31}^{\prime} x_{13}+a_{32}^{\prime} x_{23}
\end{array}\right) .
$$

Es así que las diferenciales de las flechas de grado 0 en la nueva bigráfica $\mathcal{B}^{\prime}$ han de tomarse necesariamente como las entradas de la matriz $\left(\delta^{\prime}\left(a_{i j}\right)\right):=$

$$
=\left(\begin{array}{llll}
z_{00} \alpha^{\prime} & \lambda z_{01}+a_{02} x_{21} & \lambda z_{02} & z_{02}+\lambda z_{03} \\
& & & +a_{01} x_{13}+a_{02} x_{23} \\
& & & \\
z_{10} \alpha^{\prime}-x_{13} a_{30} & \lambda z_{11}+a_{12} x_{21} & \lambda z_{12}-x_{13} a_{31} & z_{12}+\lambda z_{13} \\
& -x_{13} a_{31} & & a_{11} x_{13}+a_{12} x_{23} \\
& & & -x_{13} a_{33} \\
z_{20} \alpha^{\prime}-x_{21} a_{10} & \lambda z_{21}-x_{21} a_{11} & \lambda z_{22}-x_{21} a_{12} & z_{22}+\lambda z_{23} \\
-x_{23} a_{30} & -x_{23} a_{31} & -x_{23} a_{32} & a_{21} x_{13}+a_{22} x_{23} \\
& & & -x_{21} a_{13}-x_{23} a_{33} \\
& & & \\
z_{30} \alpha^{\prime} & \lambda z_{31}+a_{32} x_{21} & \lambda z_{32} & z_{32}+\lambda z_{33} \\
& & & +a_{31} x_{13}+a_{32} x_{23}
\end{array}\right)
$$

Por definición, $\delta^{\prime}\left(\alpha^{\prime}\right)=0, \delta^{\prime}\left(e_{j}\right)=0$, para los idempotentes $e_{j}$.

El lector puede observar ahora que las diferenciales de las flechas de grado 0 en $\mathcal{B}^{\prime}$ se definen precisamente de esta manera, a fin obtener un funtor $F: \operatorname{rep} \mathcal{B}^{\prime} \longrightarrow \operatorname{rep} \mathcal{B}$, construido 'par recollement', como se hizo en el desarrollo general de $\S 3$ supra.

El enfoque pragmático recién descrito es coherente con el punto de vista abstracto expuesto en la sección 3. Para convencerse de esto, hay que tomar $\Lambda=\{(0,0),(1,1),(2,1),(2,1)\}$, renombrando sus elementos, en el orden dado, como : $0,1,2,3$, con el fin de simplificar notaciones. Han de tomarse las matrices :

$$
g_{1}^{0}=\left(\begin{array}{cccc}
f_{0}^{0} & 0 & 0 & 0 \\
0 & f_{1}^{0} & 0 & x_{13} \\
0 & x_{21} & f_{2}^{0} & x_{23} \\
0 & 0 & 0 & f_{2}^{0}
\end{array}\right), A_{\alpha}=\left(\begin{array}{cccc}
\alpha^{\prime} & 0 & 0 & 0 \\
0 & \lambda e_{1} & 0 & 0 \\
0 & 0 & \lambda e_{2} & e_{2} \\
0 & 0 & 0 & \lambda e_{2}
\end{array}\right)
$$




$$
Y_{1}=\left(\begin{array}{cccc}
0 & 0 & 0 & 0 \\
0 & 0 & 0 & x_{13} \\
0 & x_{21} & 0 & x_{23} \\
0 & 0 & 0 & 0
\end{array}\right)
$$

La definición de las diferenciales de las flechas $z_{i j}$ de grado 1 es menos intuitiva. Para darla, obsérvese que de la condición $\delta(z)=0$ en $\mathcal{B}$, se sigue la igualdad matricial $g Z+Z g=0\left[\mathrm{Bo}_{1}\right.$, Cap. 2, 4.2] en donde $Z=\left(z_{i j}\right)$. Efectuando los productos matriciales anteriores, y despejando de manera conveniente [Ro, $\S 14 ; \mathrm{BZ}, 6.5]$, se tiene que la matriz de las diferenciales de las flechas de grado 1 es la siguiente :

$$
\begin{aligned}
& \left(\delta^{\prime}\left(z_{i j}\right)\right)=\left(\begin{array}{cccc}
f_{0}^{0} z_{00}+z_{00} f_{0}^{0} & f_{0}^{0} z_{01}+z_{01} f_{1}^{0} & f_{0}^{0} z_{02}+z_{02} f_{2}^{0} & f_{0}^{0} z_{01}+z_{03} f_{2}^{0} \\
f_{1}^{0} z_{10}+z_{10} f_{0}^{0} & f_{1}^{0} z_{11}+z_{11} f_{1}^{0} & f_{1}^{0} z_{12}+z_{12} f_{2}^{0} & f_{1}^{0} z_{13}+z_{13} f_{2}^{0} \\
f_{2}^{0} z_{20}+z_{20} f_{0}^{0} & f_{2}^{0} z_{21}+z_{21} f_{1}^{0} & f_{2}^{0} z_{22}+z_{22} f_{2}^{0} & f_{2}^{0} z_{23}+z_{23} f_{2}^{0} \\
f_{2}^{0} z_{30}+z_{30} f_{0}^{0} & f_{2}^{0} z_{31}+z_{31} f_{1}^{0} & f_{2}^{0} z_{32}+z_{32} f_{2}^{0} & f_{2}^{0} z_{33}+z_{33} f_{2}^{0}
\end{array}\right) \\
& =\left(\begin{array}{llll}
0 & & & \\
& -z_{02} x_{21} & 0 & -z_{01} x_{13}-z_{02} x_{23} \\
-x_{13} z_{30} & & & \\
& -x_{13} z_{31}-z_{12} x_{21} & -x_{13} z_{32} & -x_{13} a_{33}-a_{11} x_{13} \\
-x_{21} z_{10}-x_{23} z_{30} & -x_{21} z_{11}-x_{23} z_{31} & -x_{21} z_{12} & -x_{21} z_{13}-x_{23} z_{33} \\
+z_{20} \alpha^{\prime} & -z_{22} x_{21} & -x_{23} z_{32} & -z_{21} x_{13}-z_{22} x_{23} \\
& & & \\
0 & -z_{32} x_{21} & 0 & -z_{31} x_{13}-z_{32} x_{23} \\
0 & & &
\end{array}\right) .
\end{aligned}
$$

Parte de este trabajo aparece en la tesis doctoral del autor, escrita bajo la dirección de R. Bautista.

\section{Epílogo}

Parécele pertinente al autor compartir con quien se haya interesado en este artículo, algunas reflexiones de I. Lakatos [La, Apéndice 2, El enfoque deductivista frente al enfoque heurístico], acerca del quehacer de los matemáticos. Esto por cuanto las motivaciones que el primero tuvo al incluir el ejemplo de $\S 5$ son cercanas al espíritu que animó la valiosa crítica del húngaro. Transcripción : "La metodología euclídea ha desarrollado un cierto estilo necesario de presentación... Este estilo comienza con la enunciación de una penosa lista de axiomas, lemas y/o definiciones. Los axiomas y definiciones parecen con frecuencia artificiales y mistificadoramente complicados. Nunca se nos dice cómo surgieron esas complicaciones. La lista de axiomas y definiciones va seguida por teoremas cuidadosamente 
expresados. Estos están cargados de pesadas condiciones; parece imposible que alguien los haya barruntado alguna vez. El teorema va seguido por la prueba.

De acuerdo con el ritual euclídeo, el estudiante se ve obligado a asistir a esta conjura sin hacer preguntas ni sobre el trasfondo ni sobre cómo se realiza el juego de manos..."

"En el estilo deductivista, todas las proposiciones son verdaderas y todas las inferencias son válidas. Las matemáticas se presentan como un conjunto siempre creciente de verdades eternas e inmutables, en el que no pueden entrar los contraejemplos, las refutaciones o la crítica. El tema de estudio se recubre de un aire autoritario, al comenzar con una exclusión de monstruos disfrazada, con definiciones generadas por la prueba y con el teorema completamente desarrollado, así como al suprimir la conjetura original, las refutaciones y la crítica de la prueba. El estilo deductivista esconde la lucha y oculta la aventura. Toda la historia se desvanece, las sucesivas formulaciones tentativas del teorema a lo largo del procedimiento probatorio se condenan al olvido, mientras que el resultado final se exalta al estado de infalibilidad sagrada".

"Es muy fácil poner más ejemplos, en los que enunciar la conjetura, mostrar la prueba y los contraejemplos, siguiendo el orden heurístico hasta el teorema, y la definición generada por la prueba habría de disipar el misticismo autoritario de las matemáticas abstractas..."

\section{Referencias}

[ARS] Auslander, M.; Reiten, I.; Smalo, S. (1995) Representation Theory of Artin Algebras. Cambridge Univ. Press, Cambridge.

[BCS] Bautista, R.; Colavita, L.; Salmerón, L. (1981) "On Adjoint Funtors in Representation Theory", in Representations of Algebras, Lecture Notes in Mathematics 903, Springer-Verlag: 9-25.

[BK] Bautista, R.; Kleiner, M. (1990) "Almost split sequences for relatively projective modules", J. Algebra 135: 19-56.

[BB] Bautista, R.; Boza, J. (1996) "Reduction algorithms and quadratic forms", Public. Prelim. no. , Inst. Mat., UNAM, México.

[Bo 1 Boza, J. (1996) Algoritmos de Reducción en la Teoría de Representaciones de Coálgebras. Tesis doctoral, UNAM, México.

[Bo 2$]$ Boza, J. (1997) "Algoritmos de reducción para bigráficas diferenciales", Revista de Matemática: Teora y Aplicaciones 4(2): 24-42.

[BZ] Bautista, R.; Zuazúa, R. (1996) "Morita equivalence and reduction algorithms for representations of coalgebras", Canadian Math. Soc., Conference Proc. 18: 51-80.

[CB 1 Crawley-Boevey, W.W. (1988) "On tame algebras and bocses", Proc. London Math. Soc. 56(3): 451-483. 
$\left[\mathrm{CB}_{2}\right]$ Crawley-Boevey, W.W. (1992) "Modules of finite length over their endomorphism rings", in Representations of Algebras and Related Topics, Cambridge Univ. Press, No. 168: 127-184.

$\left[\mathrm{CB}_{3}\right]$ Crawley-Boevey, W.W. "Matrix reductions for Artinian rings, and an application to rings of finite representation type', Journal of Algebra 157(1): 1-25.

[CB 4 ] Crawley-Boevey, W.W. (1990) Matrix problems and Drozd's Theorem. Topics in Algebra, Banach Center Publ. Warzaw: 199-222.

[D $\mathrm{D}_{1}$ Drozd, Y.A. (1980) "Tame and wild matrix problems", in Representation Theory II, Lecture Notes in Mathematics 832, Springer-Verlag: 242-258.

$\left[\mathrm{D}_{2}\right]$ Drozd, Y.A. (1986) "Tame and wild matrix problems", Amer. Math. Soc. Transl. 128(2): 31-55.

[K] Kleiner, M. (1984) "Matrix problems and representations of finite dimensional algebras", Proceed. IV-ICRA, Carleton Univ., Ottawa.

[La] Lakatos, I. (1994) Pruebas y Refutaciones. La Lógica del Descubrimiento Matemático. Alianza Universidad, Madrid.

[MacL] MacLane, S. (1988) "Categories for the Working Mathematician". Springer-Verlag.

[Ma] Maltsev, A.I. (1978) Fundamentos de Álgebra Lineal. Mir, Moscú.

[M] Montaño, G. (1993) "Caracterización de bocses de dimensión finita de tipo manso". Tesis de Maestría, UNAM, México.

[O] Ovsienko, S.A. (1993) "Generic representations of free bocses", Preprint 93-03, Universität Bielefeld, $28 \mathrm{p}$.

[Ri] Ringel, C.M. (1984) Tame Algebras and Integral Quadratic Forms. Lecture Notes in Mathematics, 1099, Springer-Verlag.

[Ro] Roiter, A.V. (1980) "Matrix problems and representations of bocs's", in Lecture Notes in Mathematics 831, Springer-Verlag: 288-324.

[RK] Roiter, A.V.; Kleiner, M. "Representations of differential graded categories", in Lecture Notes in Mathematics 488, Springer-Verlag: 316-339.

[S] Simson, D. (1992) "Linear representations of partially ordered sets and vector space categories", Gordon and Breach, Switzerland. 